\title{
A hypothetical-mathematical model of acute myeloid leukaemia pathogenesis
}

\author{
Andrei Cucuianu ${ }^{\mathrm{a}}$ and Radu Precup ${ }^{\mathrm{b} *}$ \\ ${ }^{a}$ Department of Hematology, Cancer Institute, 73, 21 Decembrie Bvd, Cluj, Romania; ${ }^{b}$ Department \\ of Applied Mathematics, Babeş-Bolyai University, 400084, Cluj, Romania
}

(Received 4 February 2009; final version received 16 April 2009)

\begin{abstract}
Acute myeloid leukaemia is defined by the expansion of a mutated haematopoietic stem cell clone, with the inhibition of surrounding normal clones. Haematopoiesis can be seen as an evolutionary tree, starting with one cell that undergoes several divisions during the expansion phase, afterwards losing functional cells during the aging-related contraction phase. During divisions, offspring cells acquire 'variations', which can be either normal or abnormal. If an abnormal variation is present in more than $25 \%$ of the final cells, a monoclonal, leukemic pattern occurs. Such a pattern develops if: (A1) The abnormal variation occurs early, during the first or second divisions; (A2) The variation confers exceptional proliferative capacity; (B) A sizable proportion of the normal clones are destroyed and a previously non-significant abnormal clone gains relative dominance over a depleted environment; (C) The abnormal variation confers relative 'immortality', rendering it significant during the contraction phase. Combinations of these pathways further enhance the leukemic risk of the system. A simple mathematical model is used in order to characterize normal and leukemic states and to explain the above cellular processes generating monoclonal leukemic patterns.
\end{abstract}

Keywords: haematopoiesis; leukaemia; interacting cell populations; mathematical modelling; competitive differential system; stability

AMS Subject Classification: 92C50; 92C37; 34A34

\section{Introduction}

Haematopoiesis is usually represented as an evolutionary tree, starting with a pluripotent stem cell, a cell capable of self-renewal as well as differentiation along various pathways [41]. The stem cells undergo several divisions coupled with differentiation leading eventually to the polymorphous picture of the terminally differentiated cells found in the peripheral blood: red cells, granulocytes (neutrophils, eosinophils, basophils), monocytes, platelets, T and B lymphocytes. Stem cell differentiation is probably a random, stochastic event [45]; the fact that the final composition of the blood is relatively stable under normal conditions is the result of a regulatory process involving a complex network of positive and negative cellular and humoral factors [23] rather than the outcome of a fixed programming of the stem cells.

In fact, hematopoiesis can be viewed as an evolutionary process in an expanding population of unicellular organisms behaving along Darwinian interaction 'rules' [18].

*Corresponding author. Email: r.precup@math.ubbcluj.ro 
Within the haematopoietic microcosm, we can imagine a process in which various populations, the progeny of various stem cells, interact and 'compete'. If we view hematopoiesis as a evolutionary tree starting with one cell, it is conceivable that the daughter haematopoietic stem cells (HSC) will always display slight differences between them, variations consisting of differences in gene structure, gene expression and protein synthesis. These changes are probably more pronounced when the divisions are asymmetric, but it is highly probable that certain differences will exist even in the case of symmetric divisions, as one of the characteristics of natural systems is that there is never a complete identity between two entities, small, fractal changes being always detectable [15]. In time these differences will play against each other within the changing haematopoietic environment, leading to evolution.

Haematopoiesis displays tremendous versatility, plasticity and robustness, deriving from this apparently chaotic, stochastic process of expansion. It can be argued that the robustness of a network depends on the existence of centres of activity, which relate to a multitude of peripheral units [8]; the obliteration of a large number of peripheral units has little effect of the viability of the network while the elimination of the few 'hubs' of activity leads to its collapse. The robustness of hematopoiesis derives from the fact that HSCs may be regarded as 'hubs' of proliferative activity. It is still unclear how many HSC there are in a human being. It has been proposed that the total number of primitive HSC may be conserved across mammalian species and is about 10,000-20,000. It has been reported that in mice there is about one HSC per 30,000-50,000 nucleated marrow cells but the concentration of HSC seems to decrease in larger animals, being much lower in humans than in smaller mammals $[1,37]$. One study has even suggested that in humans, the primitive HSC concentration may be as low as 1 per 200 million marrow cells [39]. Therefore, in a normal, polyclonal hematopoiesis, destructive events involving blood cells have very little chance to actually destroy the whole haematopoietic network. It can be stated that the robustness of the haematopoietic system actually depends upon the number of stem cells, acting as hubs of normal mitotic activity, therefore the health of hematopoiesis depends on the degree of clonal diversity.

Acute myeloid leukaemia (AML) can be viewed as an evolutionary possibility, arising from the interaction and 'competition' between stem cells. Myeloid leukaemias are defined by the abnormal expansion of a single, usually mutated myeloid haematopoietic clone associated with the inhibition of the surrounding 'normal' clones [13]. It is thought that the leukemic clone starts with one cell, probably an early progenitor, the leukemic stem cell [10]. There seems to be, however, significant heterogeneity as to the levels of differentiation of leukemic stem cells. Some AML seem to occur in early, non-committed progenitors while in other cases a more differentiated progenitor is presumed [24]. Even though there is considerable heterogeneity and hierarchical organization within the leukemic clone, leukaemias are characterized by relative monotony, while normal hematopoiesis is characterized by polyclonal diversity.

The aim of this paper is to devise a simple theoretical system resembling hematopoiesis, to identify the possible general pathways through which the robustness of the system can fail, leading to leukaemia, and to give mathematical interpretation in terms of dynamic systems. The normal and leukemic states and the basic cellular processes leading to leukaemia are characterized in terms of kinetic parameters. Several mathematical models of hematopoiesis and cancer stem cells, governed by ordinary, partial or delay differential equations and systems were proposed in the last four decades (see, for example, [2,3,6,16,22,42], and [25] for a review) for a better understanding of specific biological processes, dynamic disorders and therapeutic scenarios. Our simple mathematical model was inspired by that of Dingli and 
Michor [21] and Mackey and Glass [36] and consists of a competitive, coupled differential system like those from population biology (see [11,20,38]). Under some aspects, our analysis and conclusions can be compared to those from Djulbegović-Svetina [22], where however a different system of equations of Rubinow-Lebowitz type [42] was used.

\section{The hypothetical model}

Haematopoiesis can be represented as a simple evolutionary tree, starting in a central compartment (e.g. bone marrow) with one HSC, 'A' that undergoes several divisions during the so-called expansion phase. Offspring HSCs are never completely identical, acquiring 'variations', resulting in diversity. Variations designated by Roman letters an Arabic numbers are considered 'normal'. After each division, the cells acquire a new variation. After four rounds of expansion the system has 16 more differentiated HSCs, each of them possessing five characters. The genealogical closeness between two random cells can be assessed by counting the identical and different characters (Figure 1).

We postulate that for a certain period there will exist a steady state phase, in which the 16 HSC lines will replicate/differentiate, providing the terminally differentiated cells of the peripheral compartment (e.g. peripheral blood). In this paper, we only describe what would take place in the central compartment.

Another assumption of the model is that in time, the system will suffer 'aging', gradually and randomly losing HSC lines, entering the third phase, the contraction phase. In other words, with aging the system gets poorer, less diverse (Figure 2). In fact, as HSC lines are lost, the system will develop a monoclonal pattern, usually one with normal HSC variations.

Beside the normal variations shown in Figure 1, there is the possibility of abnormal variations, designated by Greek letters and Roman numbers (Figure 3). If one single

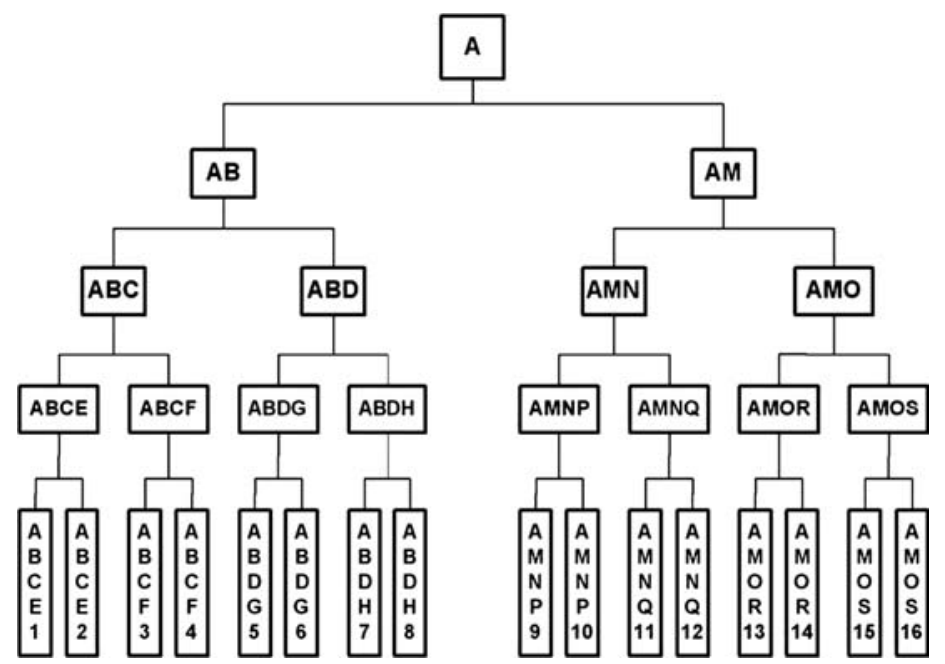

Figure 1. Normal hematopoiesis represented as an evolutionary tree starting with a primitive stem cell ' $A$ ' multiplying during the expansion phase. With each division the offspring cells acquire new variations. Variations designated by Roman letters and Arabic numbers are considered normal. Even though stem cells may actually reproduce themselves, this is not relevant here because in the definition of this model, variations, including abnormal ones, appear with each division (no two cells are completely identical, not even those cells arising from self replication). 


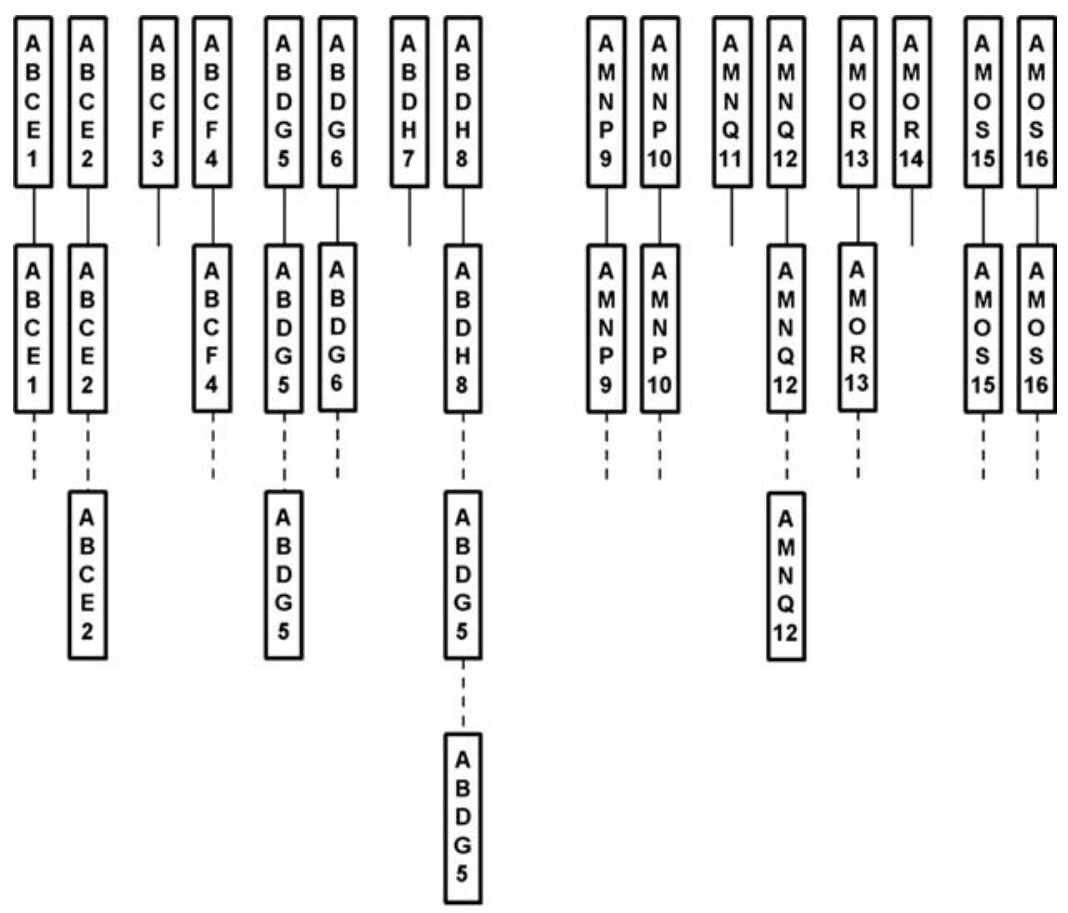

Figure 2. In time, the system suffers 'aging', gradually and randomly losing functional cell clones during the contraction phase. In other words, with aging the system gets poorer, less diverse.

abnormal variation is present in more than $25 \%$ of the final cells - a proportion similar to the $20 \%$ bone marrow blast cell percentage established as the diagnostic criteria for acute leukaemia [28] - an abnormal monoclonal leukemic pattern is present. Abnormal variations can occur at various levels in the haematopoietic tree, from a high level, involving a primitive stem cell, such as variation ' $\mu$ ', to a low, peripheral level, such as variation 'VI'.

There are several ways in which abnormal monoclonal patterns can emerge:

(A1) The presence of upstream variations (in primitive stem cells). Monoclonal patterns are obvious if the abnormal variation occurs during the first divisions as in variations ' $\mu$ ' and ' $\gamma$ '. (Figure 4).

(A2) The presence of variations that accelerate proliferation rate. If the ' $\Sigma$ ' abnormal variation confers a proliferative capacity, undergoing more divisions than normal cells, the cells bearing the ' $\Sigma$ ' variation may progress towards a monoclonal pattern and then become dominant (Figure 5).

(B) The loss of lineages and diversity in the stem cell population. If an external catastrophic event, for instance an immune or viral attack on character $\mathrm{M}$, or an intrinsic weakness of certain stem cells will wipe away at least half the final cells, the previously 'invisible', 'harmless', ' $\Phi$ ' clone, may account for more than $25 \%$ of the cells - a monoclonal pattern (Figure 6). Further events may or may not lead to a more definite 'domination' of the ' $\Phi$ ' clone. In any case, the chance of the ' $\Phi$ ' clone to acquire an opportunistic dominant role (more than 50\% of the cells) is higher now than if the other stem cell lines are present (Figure 3). 


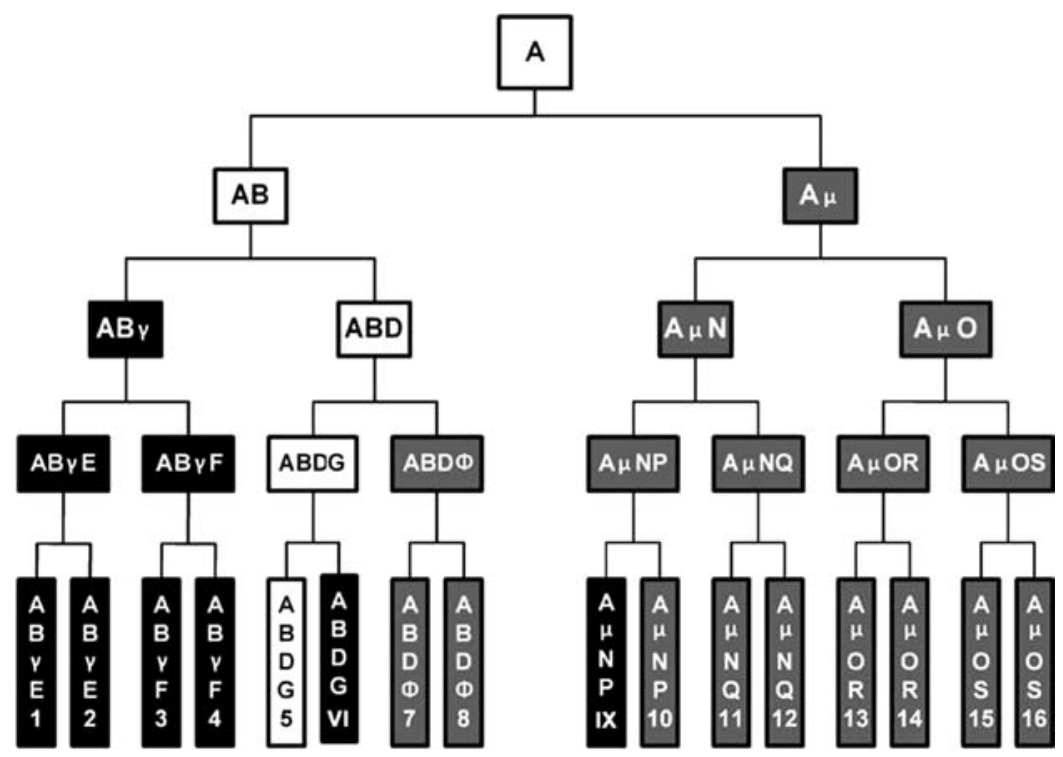

Figure 3. Divisions can also yield abnormal variations, designated by Greek letters and Roman numerals. If a certain abnormal variation is present in $\geq 25 \%$ of the final cells (similar to the diagnostic criteria for acute leukemia) - an abnormal 'monoclonal' pattern is present. Abnormal variations can occur at various levels on the hematopoietic tree.

(C) The presence of variations that prevent cell death. If the abnormal variation ' $\Omega$ ' confers 'immortality', without further enhancement, this clone has a high chance of representing more than $25 \%$ of the cells, especially during the contraction phase, eventually becoming dominant (Figure 7).

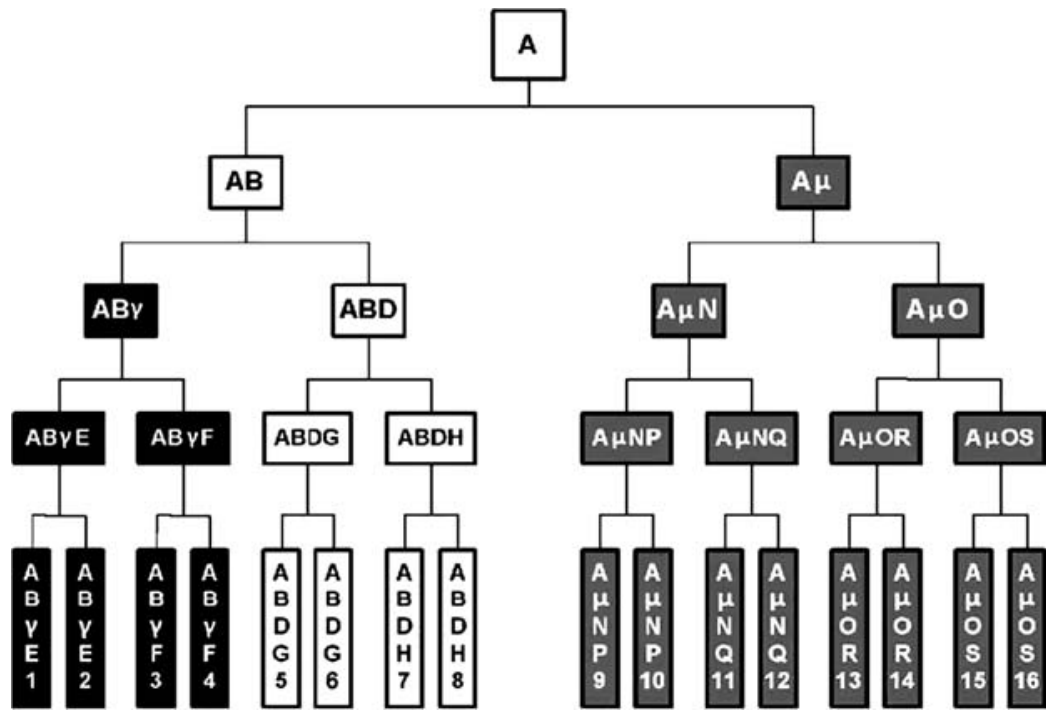

Figure 4. Leukemic pathway (A1): Monoclonal patterns are obvious if the abnormal variation occurs early, during the first divisions as in variations ' $\mu$ ', which is found in $50 \%$ of the final cells and ' $\gamma$ ', found in $25 \%$ of the cells. 


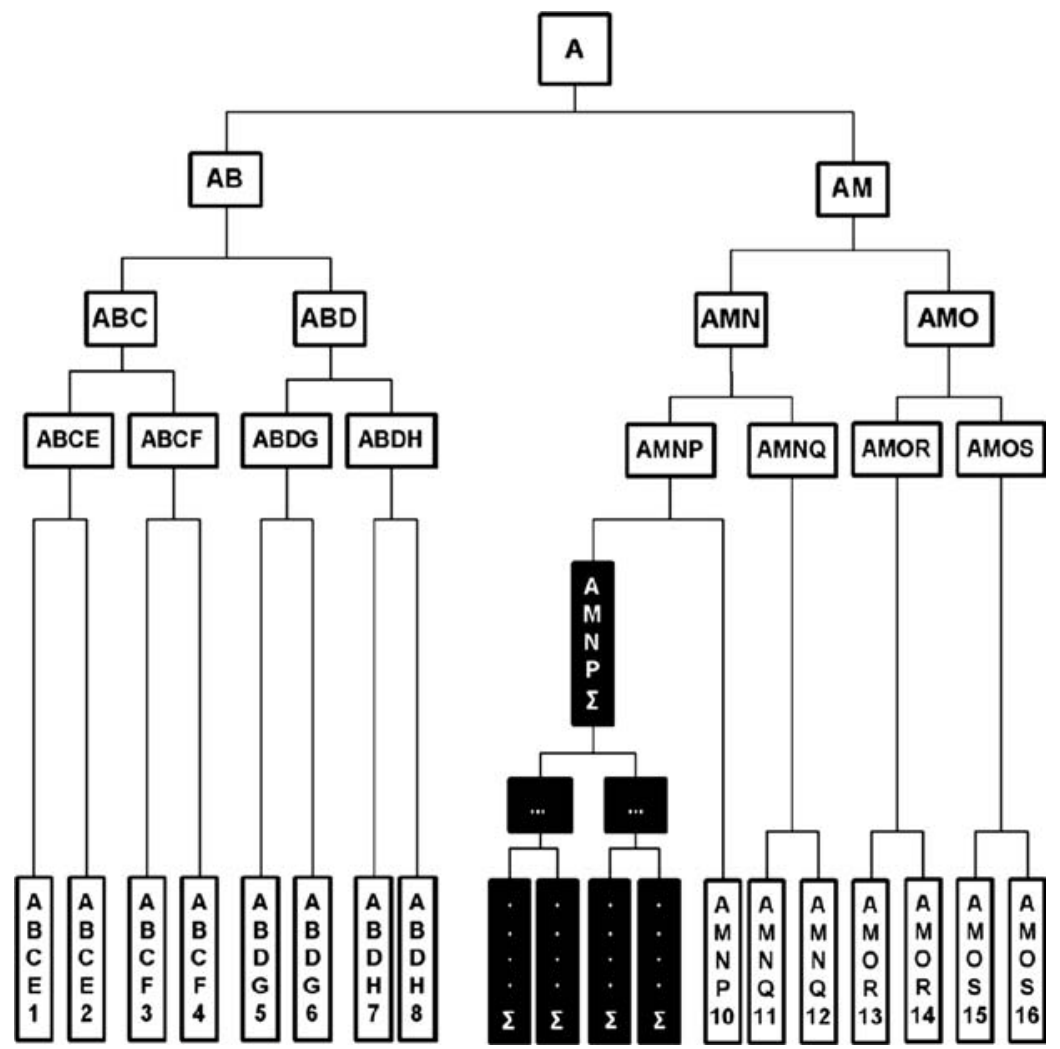

Figure 5. Leukemic pathway (A2): If the ' $\Sigma$ ' abnormal variation confers enhanced proliferative capacity, undergoing more divisions than the normal cells, the excess ' $\Sigma$ ' bearing cells may progress towards a monoclonal pattern.

Patterns (A1) and (A2) involve the existence of a 'dominant' leukemic clone, whereas in models (B) and (C) the emergence of the leukemic clone is 'opportunistic', against the background of weakness in the surrounding normal clones (see [19]).

A leukemic pattern can be the result of the above-mentioned algorithmic pathways, acting alone or in various combinations. If these four patterns can be recognized and quantified in a certain individual, an acute myeloid leukaemia risk (AMLR) can be computed. AMLR should be at its highest when all features are present, that is AMLR $=A(A 1+A 2)+B+C$.

\section{The mathematical model}

The aim of this section is to give a mathematical interpretation of the hypothetical model of acute myeloid leukemogenesis presented in Section 2. We show that there is a one-toone correspondence between cases $(\mathrm{A})-(\mathrm{C})$ from the hypothetical model and the disjoint parameter states of the differential system modelling the dynamic of the normal-leukemic cells competition. Stability of the steady states of the differential system is discussed suggesting the mathematical characterization of the normal and leukemic states and correlations between the kinetic parameters in the system are used in order to explain basic monoclonal leukemic patterns. 


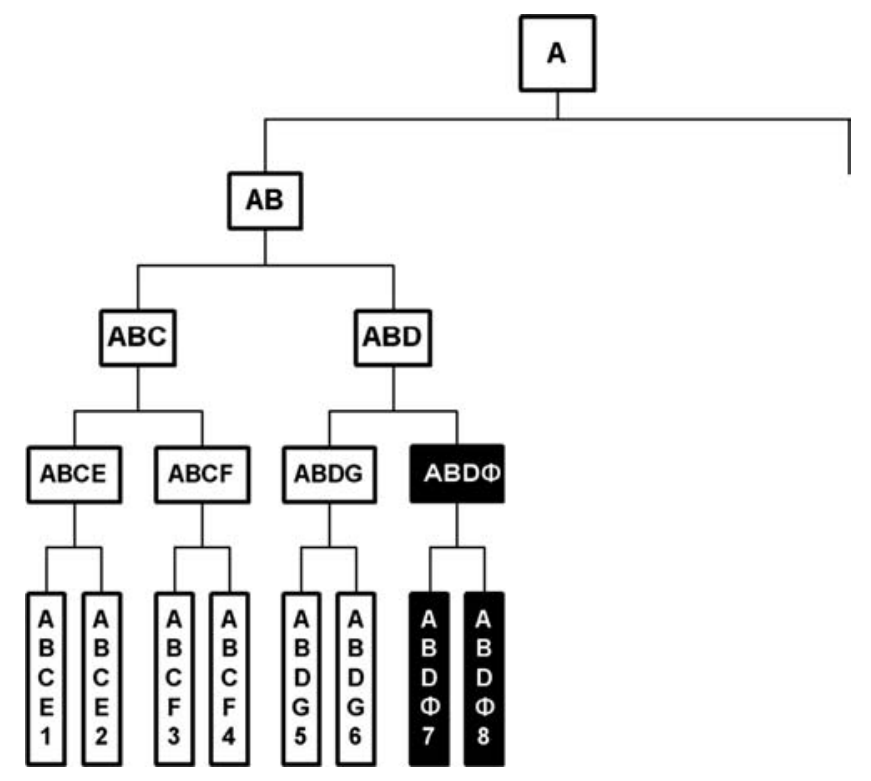

Figure 6. Leukemic pathway (B): If a catastrophic event, for instance an immune, viral, chemical or physical attack on character $\mathrm{M}$, or an intrinsic weakness of cell harbouring the $\mathrm{M}$ variation results in the disappearance of at least half the final cells, the previously non-significant ' $\Phi$ ' clone may passively account for $\geq 25 \%$ of the cells yielding a monoclonal pattern.

\subsection{The normal-leukemic dynamic system}

We shall assume that at each time $t$, the differentiated cell population divides into two: the normal population $x(t)$ and the leukemic population $y(t)$. By $x_{0}, y_{0}$, we shall denote the normal and leukemic populations at time $t=0$ (diagnostic time). We denote by $a, b, c$ and $A, B, C$ (model parameters) the growth, microenvironment sensitivity and death rates of normal and leukemic cells, respectively. The conservation laws for normal and leukemic cells can be expressed as a system of first-order differential equations (see [21,36]):

$$
\begin{aligned}
& x^{\prime}=a \frac{1}{1+b(x+y)} x-c x, \\
& y^{\prime}=A \frac{1}{1+B(x+y)} y-C y .
\end{aligned}
$$

Here $x^{\prime}=\mathrm{d} x / \mathrm{d} t$ is the time derivative which at each time gives the rate of change of population $x$, the term $(a /(1+b(x(t)+y(t)))) x(t)$ represents the new normal cells population at time $t$, and $c x(t)$ the removed normal cells at time $t$. Similar interpretations hold for $y$. The terms $1 /(1+b(x+y))$ and $1 /(1+B(x+y))$ simulate the crowding effect in the bone marrow microenvironment and introduce competition between normal and leukemic cells. Notice that the cell proliferation is faster while the total cell population $x+y$ is small, and slower for large $x+y$. Thus these terms simulate the feedback of the proliferation system. We assume that for both cell populations, the growth rate is greater than the death rate, i.e.,

$$
a>c \text { and } A>C \text {. }
$$



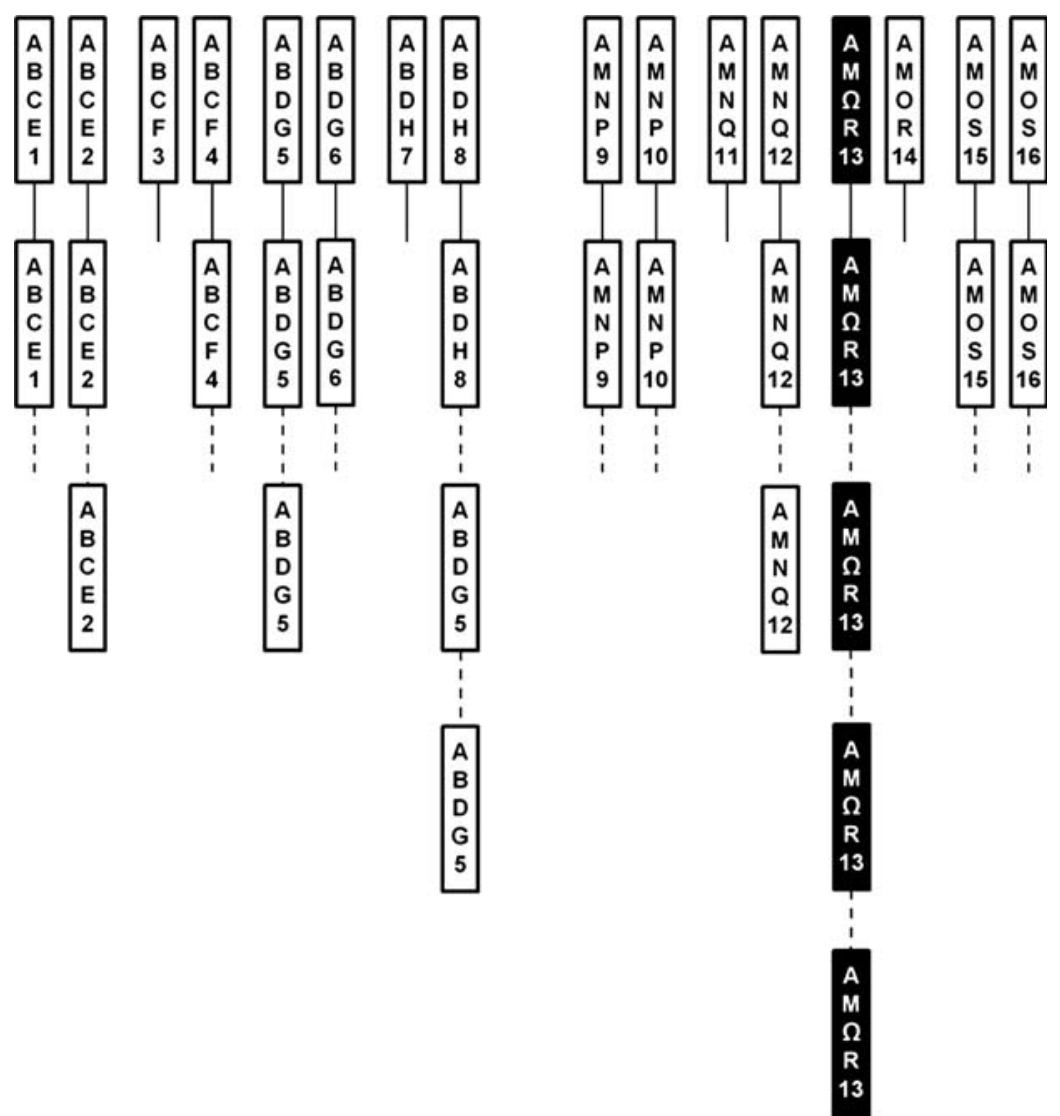

Figure 7. Leukemic pathway (C): If the ' $\Omega$ ' abnormal variation confers relative 'immortality', without further enhancement it has a high chance of appearing in more than $25 \%$ of the cells during the contraction phase, eventually becoming dominant.

Denote

$$
d:=\frac{1}{b}\left(\frac{a}{c}-1\right) \text { and } D:=\frac{1}{B}\left(\frac{A}{C}-1\right)
$$

Here are some basic properties which can be deduced from system (1):

(1) Monotonicity of solutions. Normal cell population grows, that is $x^{\prime}(t)>0$, as long as $a /(1+b(x+y))-c>0$, i.e., $x+y<d$, and decreases if $x+y>d$. Similarly, $y$ grows as long as $x+y<D$ and decreases if $x+y>D$.

(2) Steady-states. Steady-states or equilibria are constant solutions, i.e., solutions with $x^{\prime}=0, y^{\prime}=0$. If $d \neq D$, then the steady-states are:

$$
(0,0) ;(d, 0) ;(0, D) \text {. }
$$

If $d=D$, then the equilibria are:

$$
(0,0) ;(\alpha, d-\alpha) \text { for } 0 \leq \alpha \leq d \text {. }
$$


(3) Stability. Recall (see, for example, $[4,14])$ that an equilibrium $(\alpha, \beta)$ of the differential system

$$
\begin{aligned}
& x^{\prime}=f(x, y), \\
& y^{\prime}=g(x, y),
\end{aligned}
$$

i.e. a solution of the algebraic system $f(\alpha, \beta)=0, g(\alpha, \beta)=0$, is said to be stable if small changes at any given time $t_{0}$ bring only small changes in the solutions for all $t \geq t_{0}$. Otherwise, we say that the solution $(\alpha, \beta)$ is unstable. Also, $(\alpha, \beta)$ is said to be asymptotically stable if it is stable and in addition $x(t)$ tends to $\alpha$ and $y(t)$ tends to $\beta$ as $t$ goes to infinity, whenever $x\left(t_{0}\right), y\left(t_{0}\right)$ are close enough to $\alpha, \beta$, respectively. It is well known [4, Chapter 24], [14, Chapter 13] that $(\alpha, \beta)$ is asymptotically stable if the Jacobian matrix $J(\alpha, \beta)$ is Hurwitz, i.e., $\operatorname{Re} \lambda<0$ for all its characteristic roots $\lambda$, and unstable if $\operatorname{Re}$ $\lambda>0$ for at least one of its characteristic roots. Recall that the Jacobian matrix $J(x, y)$ is defined by

$$
\left[\begin{array}{ll}
f_{x}^{\prime}(x, y) & f_{y}^{\prime}(x, y) \\
g_{x}^{\prime}(x, y) & g_{y}^{\prime}(x, y)
\end{array}\right]
$$

Using these criteria, we can show that the solution $(0,0)$ is unstable under our assumption (2). Also

$$
\begin{aligned}
& (d, 0) \text { is asymptotically stable and }(0, D) \text { is unstable, if } d>D ; \\
& (d, 0) \text { is unstable and }(0, D) \text { is asymptotically stable, if } d<D .
\end{aligned}
$$

The case of the equality $d=D$ is particularly interesting from the point of view of stability. For example, in case that $a=A, b=B$ and $c=C$, when $d=D$ as well, if we add the equations in (1), we obtain the equation of $x+y$, namely

$$
(x+y)^{\prime}=\left(a \frac{1}{1+b(x+y)}-c\right)(x+y) .
$$

For this equation, the steady-state $x+y=d$ is asymptotically stable. Also, if we multiply the first equation in (1) by $y$, and the second equation by $x$ and we substrate, we obtain

$$
\left(\frac{x}{y}\right)^{\prime}=0
$$

which shows that the ratio $x / y$ is constant in time. Hence $y=\gamma x$ for $\gamma=y_{0} / x_{0}$, and if we substitute into the first equation of (1) we obtain the equation of $x$, namely

$$
x^{\prime}=a \frac{1}{1+b(1+\gamma) x} x-c x .
$$

Notice that the positive steady-state of this equation $\alpha:=d /(1+\gamma)$ is asymptotically 
stable. Consequently, the equilibrium

$$
(\alpha, d-\alpha) \text { is stable for every } 0<\alpha<d .
$$

\subsection{Numerical simulation}

We shall simulate numerically system (1) using the Maple package, version 11. Graphs of $x(t)$ (broken line) and $y(t)$ (solid line) for $0 \leq t \leq 100$, are represented for different values of the model parameters $a, b, c, A, B, C$ and initial values $x(0)$ and $y(0)$. For example, if $a:=0.3 ; b:=0.5 ; c:=0.1 ; A:=0.3 ; B:=0.5 ; C:=0.1 ; x(0)=4.5$ and $y(0)=1.5$, we use the following sequence of commands:

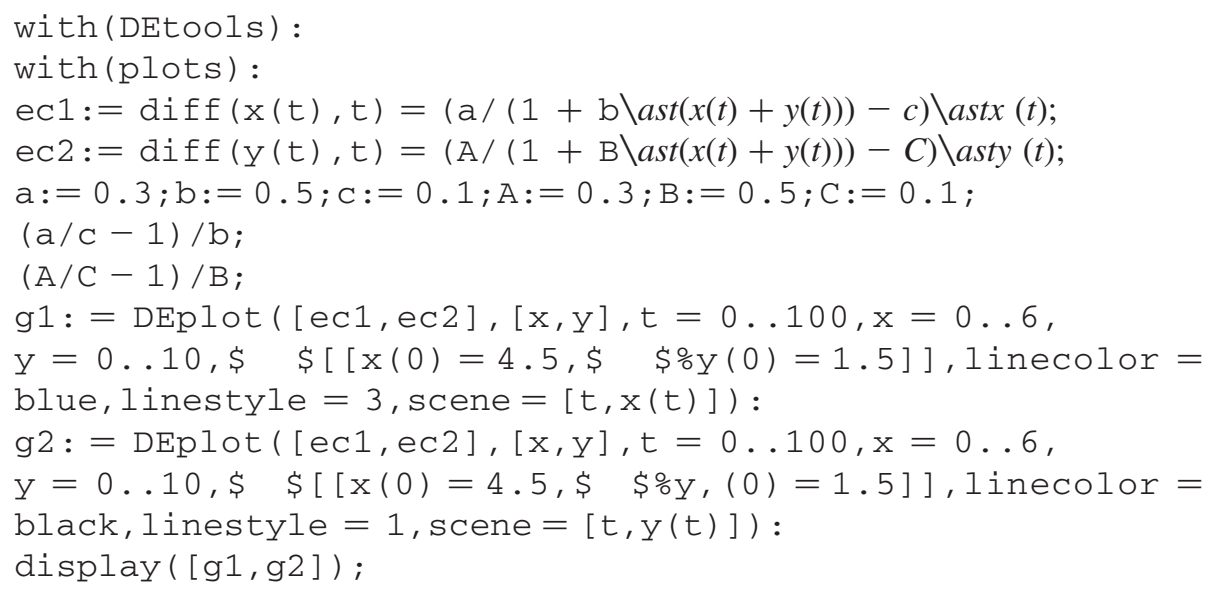

The aim of our numerical simulations confines to the understanding of the dynamic behaviour of the mathematical system. In this respect, we use fictive values of parameters and not clinical, experimental ones, although such values are available in the literature [21].

\subsection{Mathematical basis of leukemogenesis}

The stability analysis in Section 3.1 shows that there is only one asymptotically stable steady-state of the system, namely $x^{*}=d, y^{*}=0$ (no leukemic cells) if $d>D$. Therefore, we may say that the case $d>D$ corresponds to a healthy haematopoietic system or to the eradication of the disease. Thus leukemic cells exist only if $d \leq D$. We already know that $d<D$ implies the unique asymptotically stable steady-state $x^{*}=0$ (no normal cells), $y^{*}=D$ (leukemic cells only), hence the case $d<D$ corresponds to leukemic diseases.

Finally, the equality $d=D$ allows the coexistence of normal and malignant cell populations around different equilibria of the type $x^{*}=\alpha, y^{*}=d-\alpha$, depending on the initial conditions $x_{0}, y_{0}$. Thus, if the leukemic cell population $y(t)$ is caused by an abnormal mutation (a mild clone) which does not distort the basic cell parameters: growth, sensitivity and death rates, i.e., $a=A, b=B, c=C$, and consequently, $d=D$, then the normal and abnormal cell populations $x, y$ will coexist (Figure 8) under the constant proportion $\kappa:=x_{0} / y_{0}$, the total cell population $x+y$ tends to $d, x$ tends to $\alpha$ and $y$ to $d-\alpha$, for some $\alpha$ depending on initial concentrations $x_{0}, y_{0}$. More exactly, from $x / y=\kappa$, we obtain $\alpha /(d-\alpha)=\kappa$, whence $\alpha=\kappa d /(1+\kappa)$.

However, a condition like $d=D$ is physiologically very unstable since no matter how small the variations of kinetic parameters, they can switch it into the leukemic state $d<D$. 
(a)

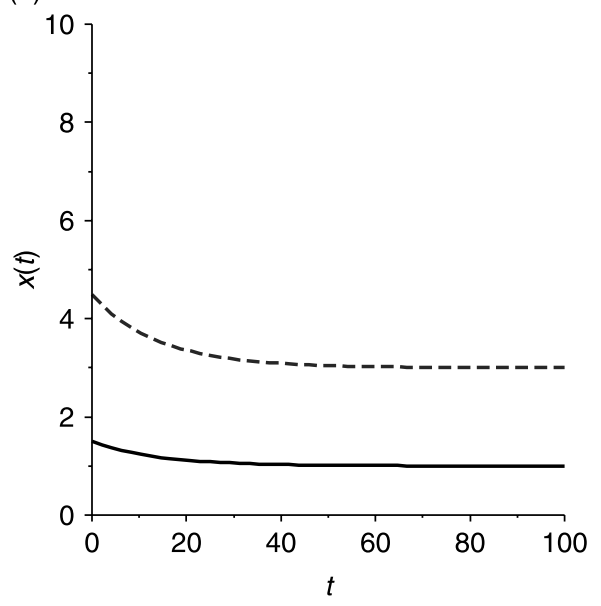

(b)

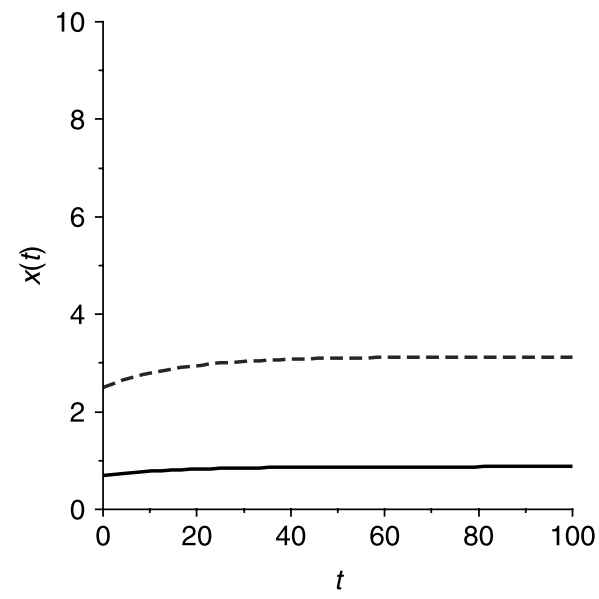

Figure 8. Numerical simulations for the coexistence case $d=D$. Here $a=A=0.3 ; b=B=0.5$; $c=C=0.1$, and $d=D=4$. The normal cell population $x$ (broken line) and the abnormal cell population $y$ (solid line) will coexist under the constant proportion $\kappa:=x_{0} / y_{0}$. Two cases are considered: (a) $x_{0}=4.5>d, y_{0}=1.5<d, x_{0}+y_{0}>d$ and (b) $x_{0}=2.5<d, y_{0}=0.7<d$, $x_{0}+y_{0}<d$.

Our next goal is to explain the four basic pathways leading to leukaemia which were described in Section 2, and their corresponding monoclonal patterns, by the alteration of a single one of the kinetic parameters $a, b, c$ in (1). In our model, the leukemic state being characterized by the inequality $d<D$, three cases are mathematically possible: $a<$ $A ; b>B$ and $c>C$.

CASE (A1). We assume that the abnormal variation occurring during the first divisions does not change neither the interval between divisions and nor sensibility and death parameters. Hence $b=B$ and $c=C$. A monoclonal pattern is then perceptible if at a given time $T$ the ratio $y(T) / x(T)$ between abnormal and normal cells becomes greater than the initial (at time 0) ratio $y_{0} / x_{0}$. From (1), we have

$$
\begin{aligned}
\frac{x^{\prime}}{x} & =\frac{a}{1+b(x+y)}-c, \\
\frac{y^{\prime}}{y} & =\frac{A}{1+b(x+y)}-c .
\end{aligned}
$$

Integration from 0 to $T$ gives

Consequently,

$$
\begin{aligned}
& \ln \frac{x(T)}{x_{0}}=\int_{0}^{T}\left(\frac{a}{1+b(x+y)}-c\right) \mathrm{d} t, \\
& \ln \frac{y(T)}{y_{0}}=\int_{0}^{T}\left(\frac{A}{1+b(x+y)}-c\right) \mathrm{d} t .
\end{aligned}
$$

$$
\ln \frac{y(T)}{y_{0}}-\ln \frac{x(T)}{x_{0}}=(A-a) \int_{0}^{T} \frac{1}{1+b(x+y)} \mathrm{d} t
$$


or, equivalently,

$$
\ln \frac{y(T)}{x(T)} \frac{x_{0}}{y_{0}}=(A-a) \int_{0}^{T} \frac{1}{1+b(x+y)} \mathrm{d} t .
$$

This together with $(y(T) / x(T))>y_{0} / x_{0}$ implies that $A-a>0$ (Figure 9), i.e. cancer cells have a larger proliferation rate than normal cells. Notice that for small $x+y$ formula (3) gives

$$
A-a \simeq \frac{1}{T} \ln \frac{y(T)}{x(T)} \frac{x_{0}}{y_{0}}
$$

a measure of 'aggressivity' of the cancer cells.

CASE (A2). An accelerated proliferation rate can also be put in connection with a shortened interval between divisions. Thus, in our model, pathway (A2) is also characterized by the parameter inequality $a<A$ (Figure 9).

CASE (B). Monoclonal patterns caused by external catastrophic events or intrinsic weakness of certain stem cells can be explained by the change of sensibility of particular clones. Thus, we put case (B) in connection with the sensitivity parameter inequality $b>B$ (Figure 10).

Case (C). Dominant clones due to abnormal variations conferring 'immortality' are characterized by a lower death rate. Thus case $(\mathrm{C})$ is mathematically expressed by the

(a)

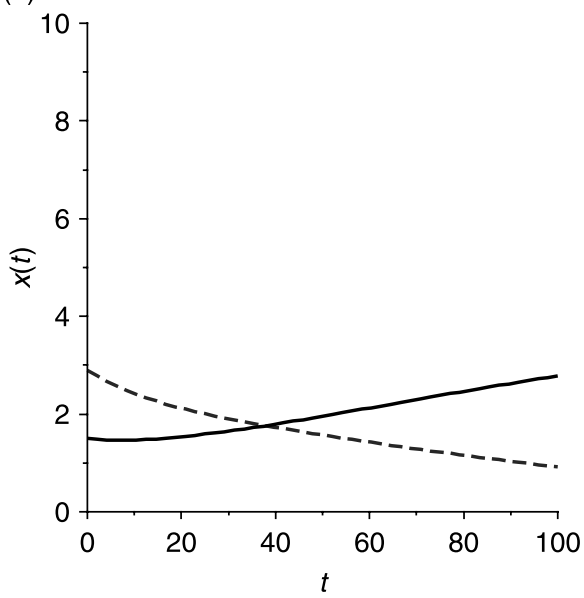

(b)

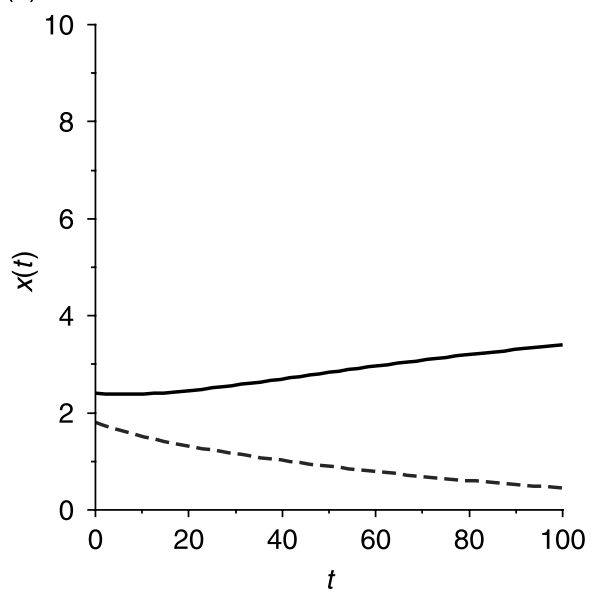

Figure 9. Numerical simulations for the case $a<A$. Here, $a=0.25$; $A=0.3 ; b=B=0.5 ; c=C=0.1, d=3$ and $D=4$. In time, the normal cell population $x(t)$ (broken line) approaches 0 while the abnormal cell population $y(t)$ (solid line) becomes arbitrarily close to the value $D$. Two cases are considered: (a) $x_{0}=2.9, y_{0}=1.5$ and (b) $x_{0}=1.8, y_{0}=2.4$. 
inequality $C<c$. Notice that, as in case (A1), we can obtain the estimation

a measure of the 'immortality' of the clone.

$$
c-C=\frac{1}{T} \ln \frac{y(T)}{x(T)} \frac{x_{0}}{y_{0}},
$$

In conclusion, each one of basic cases $(\mathrm{A}-\mathrm{C})$ leading to leukaemia corresponds to the alteration of a single kinetic parameter: thus (A) corresponds to an increased growth rate $A>a$; (B) to a decreased sensibility rate $B<b$ and (C) to a decreased death parameter $C<c$. Obviously, a monoclonal pattern becomes more prominent when two of these alterations or all three of them hold. More complex leukemic patterns are due to the proliferation of several (say $n$ ) abnormal clones with their own alterations of the kinetic parameters. Mathematically, this could be described by an $(n+1)$-dimensional competitive system whose parameters are $a, b, c$ (for normal cells) and $A_{i}, B_{i}, C_{i}(i=$ $1,2, \ldots, n)$ (for each of the abnormal clones). Then the dynamic of the whole system depends on the own parameter modifications of the clones.

\section{Conclusions}

\subsection{Some clinical remarks}

The models that we have outlined could be extrapolated to real leukaemia cases. Theoretically, all terms can be quantified; practically there probably are still too many unknown parameters to devise a clinically useful mathematical model. However, there are some clues as to the applicability of these patterns, deriving from the epidemiological picture in various patient populations.

1. In the general population, AMLR is low, amounting to approximately 4$5 / 100,000 / y e a r$ [13]. In fact, AML is quite a rare oncological event probably due to the robustness of the haematopoietic system, conferred by its rich clonal diversity.

(a)

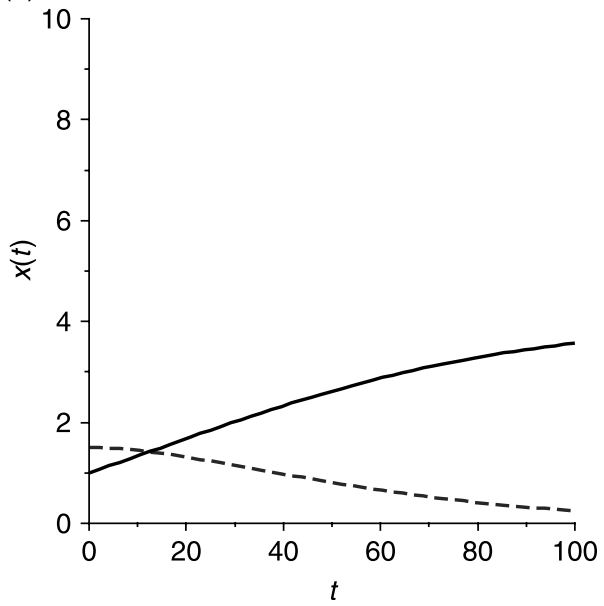

(b)

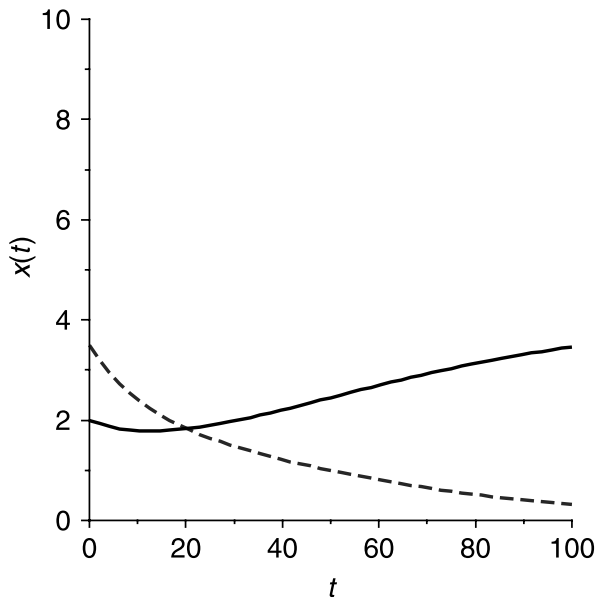

Figure 10. Numerical simulations for the case $b>B$. Here $a=A=0.3 ; b=0.8 ; B=0.5$; $c=C=0.1$, when $d=2.5, D=4$. In time, the normal cell population $x(t)$ (broken line) approaches 0 while the abnormal cell population $y(t)$ (solid line) becomes arbitrarily close to the value $D$. Two cases are considered: (a) $x_{0}=1.5, y_{0}=1$ and (b) $x_{0}=3.5, y_{0}=2$. 
However, even in the general population there are marked differences in AML incidence, according to age. The incidence is very low, about $1 / 100,000 /$ year under 30 years, and much higher, 10-20/100,000/year in subjects over 60 years. It is possible that in some older subjects the clonal diversity may weaken, as suggested by a study showing an increase of blood monoclonal patterns with aging [12]. These findings were not confirmed in a more recent study [44], and it seems that the absolute number of stem cells does not decrease significantly with age. However, it was shown that hematopoiesis is functionally disregulated in aging subjects, resulting in reduced bone marrow cellularity and impaired response to stimuli [26]. Therefore, in old subjects, pattern (C) may play an important role, while in young subjects, it takes a highly proliferative clone to become dominant, such as in model (A2).

2. In patients with myelodysplastic syndromes (MDS), AMLR is high, 5-50\% of all cases progressing to overt AML [5]. MDS occurs mostly in old subjects and is characterized by clonal, ineffective hematopoiesis. MDS progenitors display an increased propensity towards cell death through apoptosis, leading to ineffective hematopoiesis [40]. It is conceivable that against this background of widespread apoptosis, even a slow-proliferating leukemic clone, resistant to apoptosis, relatively 'immortal', might gain proliferative advantage and eventually become dominant. Therefore, in MDS, AMLR could be enhanced in an opportunistic manner through a combination of patterns (B) (intrinsic defect of the non-leukemic clones) and (C) (old age).

3. In subjects previously treated with chemo/radiotherapy, AMLR is high. For instance, the risk of treatment-related AML and MDS in non-Hodgkin's lymphoma is $10 \%$ within 10 years of primary therapy, a much higher risk than that observed in the general population [7]. One can assume that alkylating agents and radiotherapy, aside from increasing the variation rate, also lead to the destruction of a large number of HSC lines, such as in model B. The same pattern may apply to AML following hereditary disorders associated with marrow aplasia such as Fanconi's anaemia or Bloom's syndrome [32,34] in which the depletion of normal progenitors creates the background in which a 'sluggish' leukemic clone can emerge.

4. In chronic myelogenous leukaemia, (CML), AMLR is high, practically $100 \%$ of the patients progressing to AML [17]. CML in chronic phase is best described by pattern (A1), with variation ' $\mu$ ' as $\mathrm{BCR}-\mathrm{ABL}$ and additional variation 'IX' related to clonal evolution leading to the AML-like blastic phase. Actually in CML, blastic phase may be the result of a combination of cases (A1) and (A2).

5. Pattern (A2) may describe best the so-called 'true de novo' AML, such as the corebinding factor (CBF) mutated AML, the NPM1 positive AML or the CBPApositive AMLs, which generally occur in younger patients and the mutations appear in more differentiated progenitor cells, leading to a dominant pattern through enhanced proliferation [30,35].

\subsection{Implications for therapy}

In leukaemia, therapy should aim at reversing the monoclonal pattern. Targeted therapy, meaning the specific removal or repair of the leukemic variation is still not feasible in most AML patients, even though incriminating genetic variations can nowadays be found in a significant proportion of patients [43]. Even if targeted therapies do become available in the future, they have the highest chance of success in models (A1) and (A2), 
where there is an active proliferation. In models (B) and (C) targeted therapy would not reverse the poverty and instability of the system. Presently, aggressive chemotherapy is the main weapon in AML. However, chemotherapy is often ineffective against primitive stem cells, which frequently include leukemic HSC [27]. Therefore, conventional aggressive chemotherapy is best suited for model (A2). In model (A1) it leaves the variation in place and in models (B) and (C), chemotherapy may only worsen the intrinsic poverty of the system. This assumption is somewhat supported by the poor results obtained with chemotherapy in older AML subjects and in secondary and MDSrelated AML [33]. Allogeneic HSC transplantation (alloHSC) is still the best therapy in AML, especially in high-risk cases [46]. Theoretically, it may best be suited for models (A1), (B) and (C), where normal hematopoiesis is intrinsically deficient and has to be replaced or 'rejuvenated'. One notable example is the fact that alloHSC remains the only curative treatment in CML, despite the advent of the relatively targeted treatment represented by tyrosine kinase inhibitors (TKI). Even though TKI are more successful in controlling the disease than previous therapies, they rarely lead to complete molecular remissions, in other words they do not eradicate the CML stem cells [9,31]. Unfortunately alloHSC transplantation is associated with a much higher rate of procedure-related mortality and morbidity in older subjects than in younger ones. AlloHSC may not be needed in AML described by pattern (A2), in which the mutations occur in more differentiated HSC. Indeed, 'true de novo' AML, such as CBF-mutated AML, CBPA and NPM1 mutated AML, have a good prognosis with conventional chemotherapy alone [29,35].

In conclusion, there is a finite number of ways in which a genealogical tree-like system resembling hematopoiesis can acquire a monoclonal, leukemic pattern. A mutated clone may become dominant either actively, through variations conferring increased proliferation potential, or opportunistically, taking advantage of a damaged or aging normal clone compartment. Therapeutic strategies should take into account these profiles in individual leukaemia patients.

\section{Acknowledgement}

The authors would like to thank Professors Damian Trif and Marcel Serban for their help during the preparation of the manuscript. They are also grateful to the referee whose comments and suggestions have led to an improvement of the biology part of the paper.

\section{References}

[1] J.L. Abkowitz, S.N. Catlin, M.T. McCallie, and P. Guttorp, Evidence that the number of haematopoietic stem cells per animal is conserved in mammals, Blood 100 (2002), pp. 2665-2667.

[2] M. Adimy, F. Crauste, and A. El Abdllaoui, Discrete maturity-structured model of cell differentiation with applications to acute myelogenous leukaemia, J. Biol. Systems 16 (2008), pp. 395-424.

[3] E.K. Afenya and D.E. Bentil, Some perspectives on modeling leukaemia, Math. Biosci. 150 (1998), pp. 113-130.

[4] R.P. Agarwal and D. O'Regan, An Introduction to Ordinary Differential Equations, Springer, Berlin, 2008.

[5] M. Albitar, T. Manshouri, and Y. Shen, Myelodysplastic syndrome is not merely 'preleukaemia', Blood 100 (2002), pp. 791-798.

[6] L.K. Andersen and M.C. Mackey, Resonance in periodic chemotherapy: a case study of acute myelogenous leukaemia, J. Theor. Biol. 209 (2001), pp. 113-130.

[7] J.O. Armitage, P.P. Carbone, and J.M. Connors, Treatment related myelodysplasia and acute leukaemia in non-Hodgkin's lymphoma patients, J. Clin. Oncol. 21 (2002), pp. 897-906. 
[8] A.L. Barabassi and Z.N. Oltvai, Network biology: understanding the cell's functional organization, Nat. Rev. Genet. 5 (2004), pp. 101-113.

[9] R. Bhatia, M. Holtz, and N. Niu, Persistence of malignant haematopoietic progenitors in chronic myelogenous leukaemia patients in complete cytogenetic remission following imatinib mesylate treatment, Blood 101 (2003), pp. 4701-4708.

[10] D. Bonnet, Normal and leukemic CD34 negative human haematopoietic stem cells, Rev. Clin. Exp. Hematol. 5 (2001), pp. 42-61.

[11] F. Brauer and C. Castillo-Chávez, Mathematical Models in Population Biology and Epidemiology, Springer, Berlin, 2001.

[12] L. Busque, R. Mio, J. Mattioli, E. Brais, N. Blais, Y. Lalonde, M. Maragh, and D.G. Gilliland, Nonrandom $X$-inactivation patterns in normal females: lyonization ratios vary with age, Blood 88 (1996), pp. 59-65.

[13] C.A. Clarke and S.L. Glaser, Acute myeloid leukaemia, N. Eng. J. Med. 342 (2000), pp. $358-361$.

[14] E.A. Coddington and N. Levinson, Theory of Ordinary Differential Equations, Tata McGrawHill, New Delhi, 1972.

[15] D.S. Coffey, Self-organization, complexity and chaos: The new biology for medicine, Nat. Med. 4 (1998), pp. 882-885.

[16] C. Colijn and M.C. Mackey, A mathematical model of hematopoiesis-I. Periodic chronic myelogenous leukaemia, J. Theor. Biol. 237 (2005), pp. 117-132.

[17] J. Cortes and M.E. O'Dwyer, Clonal evolution in chronic myelogenous leukaemia, Hematol. Oncol. Clin. North Am. 18 (2004), pp. 671-684.

[18] A. Cucuianu, Cell darwinism, apoptosis, free radicals and haematological malignancies, Med. Hypotheses 56 (2001), pp. 52-57.

[19] A. Cucuianu, Dominant and opportunistic leukemic clones: proposal for a pathogenesisoriented classification in acute myeloid leukaemia, Med. Hypotheses 65 (2005), pp. 107-113.

[20] O. Diekmann, R. Durrett, K.P. Hadeler, P. Maini, and H.L. Smith, Mathematics Inspired by Biology, Springer, Berlin, 1999.

[21] D. Dingli and F. Michor, Successful therapy must eradicate cancer stem cells, Stem Cells 24 (2006), pp. 2603-2610.

[22] B. Djulbegović and S. Svetina, Mathematical model of acute myeloblastic leukaemia: an investigation of the relevant kinetic parameters, Cell Proliferation 18 (1985), pp. 307-319.

[23] C.J. Eaves, J.D. Cashman, H.J. Sutherland, T. Otsuka, R.K. Humphries, D.E. Hogge, P.L. Lansdorp, and A.C. Eaves, Molecular analysis of primitive haematopoietic cell proliferation control mechanisms, Ann. NY Acad. Sci. 628 (1991), pp. 298-306.

[24] P.J. Fialkow, J.W. Singer, and W.H. Raskind, Clonal development, stem-cell differentiation and clinical remission in acute non-lymphocytic leukaemia, N. Eng. J. Med. 317 (1987), pp. $468-473$.

[25] C. Foley and M.C. Mackey, Dynamic hematological disease: a review, J. Math. Biol. 58 (2009), pp. 285-322.

[26] R. Gazit, I.L. Weissman, and D.J. Rossi, Haematopoietic stem cells and the aging haematopoietic system, Semin. Hematol. 45 (2008), pp. 218-224.

[27] J. Griffin and B. Lowenberg, Clonogenic cells in acute myeloblastic leukaemia, Blood 68 (1986), pp. 1185-1195.

[28] N.L. Harris, E.S. Jaffe, J. Diebold, G. Flandrin, H.K. Muller-Hermelink, J. Vardiman, T.A. Lister, and C.D. Bloomfield, The World Health Organization Classification of Neoplasms of the Haemopoietic and Lymphoid Tissues: report of the Clinical Advisory Comitee MeetingAirlie House, Virginia, November 1997, Hematol. J. 1 (2000), pp. 53-66.

[29] S.M. Hart and L. Foroni, Core binding factor genes and human leukaemia, Haematologica 87 (2002), pp. 1307-1323.

[30] D.R. Head, Revised classification of acute leukaemias, Leukemia 10 (1996), pp. 1826-1831.

[31] E. Jabbour, J.E. Cortes, and H.M. Kantarjian, Molecular monitoring in chronic myeloid leukaemia. Response to tyrosine kinase inhibitors and prognostic implications, Cancer 15 (2008), pp. 2112-2118.

[32] H. Kaneko and N. Kondo, Clinical features of Bloom syndrome and function of the causative gene, BLM helicase, Expert Rev. Mol. Diagn. 4 (2004), pp. 393-401.

[33] C.P. Leith, K.J. Kopecky, and J. Goodwin, Acute myeloid leukaemia in the elderly: assessment of multidrug resistance and cytogenetics distinguishes biologic subgroups with remarkable 
distinct responses to standard chemotherapy. A Southwest Oncology Group Study, Blood 89 (1997), pp. 3323-3328.

[34] M.W. Lensch, R.K. Rathburn, and S.B. Olson, Selective pressure as an essential force in molecular evolution of myeloid leukemic clones: a view from the window of Fanconi anemia, Leukemia 13 (1999), pp. 1784-1789.

[35] B. Löwenberg, Acute myeloid leukaemia: The challenge of capturing disease variety, Hematology Am. Soc. Hematol. Educ. Program (2008), pp. 1-11.

[36] M.C. Mackey and L. Glass, Oscillation and chaos in physiological control systems, Science 197 (1977), pp. 287-289.

[37] K.F. McCarthy, Marrow frequency of long-term repopulating cells: evidence that marrow haematopoietic cell concentration may be inversely proportional to species body weight, Blood 101 (2003), pp. 3431-3436.

[38] J.D. Murray, Mathematical Biology II: Spatial Models and Biomedical Applications, Springer, Berlin, 2003.

[39] R. Nash, R. Storb, and P. Neiman, Polyclonal reconstitution of human marrow after allogeneic bone marrow transplantation, Blood 72 (1988), pp. 2031-2037.

[40] F. Nolte and W.K. Hofmann, Molecular pathogenesis and genomic changes, Ann. Hematol. 10 (2008), pp. 777-795.

[41] C. Peschle, R. Botta, R. Muller, M. Valtieri, and B. Ziegler, Purification and functional assay of pluripotent haematopoietic stem cells, Rev. Clin. Exp. Hematol. 5 (2001), pp. 3-14.

[42] S.I. Rubinow and J.L. Lebowitz, Model of cell kinetics with applications to the acute myeloblastic leukaemia state in man, Biosystems 8 (1977), p. 265.

[43] R.F. Schlenk, K. Döhner, J. Krauter, S. Fröhling, A. Corbacioglu, L. Bullinger, M. Habdank, D. Späth, M. Morgan, A. Benner, B. Schlegelberger, G. Heil, A. Ganser, H. Döhner and GermanAustrian Acute Myeloid Leukemia Study Group, Mutations and treatment outcomes in cytogenetically normal acute myeloid leukaemia, N. Engl. J. Med. 358 (2008), pp. 1909-1918.

[44] S.I. Swierczek, N. Agarwal, R.H. Nussenzveig, G. Rothstein, A. Wilson, A. Artz, and J.T. Prchal, Haematopoiesis is not clonal in healthy elderly women, Blood 112 (2008), pp. 3186-3193.

[45] I. Thornley, R. Sutherland, R. Wynn, R. Nayar, L. Sung, G. Corpus, T. Kiss, J. Lipton, J. Doyle, F. Saunders, S. Kamel-Reid, M. Freedman, and H. Messner, Early haematopoietic reconstitution after clinical stem cell transplantation: evidence for stochastic stem cell behavior and limited acceleration in telomere loss, Blood 99 (2002), pp. 2387-2396.

[46] A. Wahlin, B. Markevarn, I. Golovleva, and M. Nilsson, Improved outcomes in AML are restricted to young patients and are related mostly to bone marrow transplantation therapy, Eur. J. Hematol. 68 (2002), pp. 232-239. 


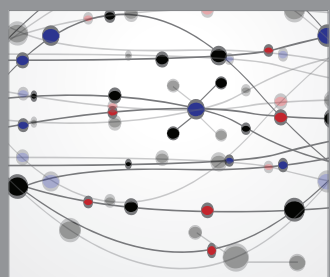

The Scientific World Journal
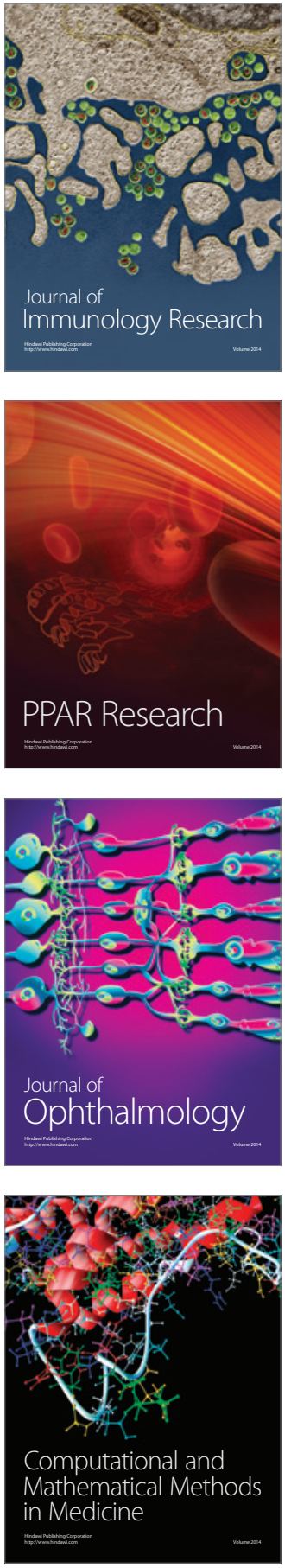

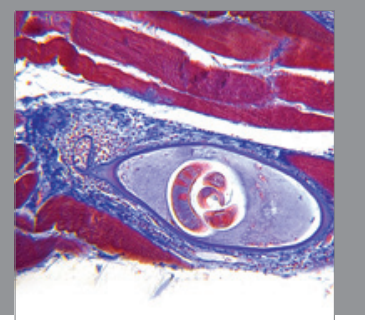

Gastroenterology

Research and Practice
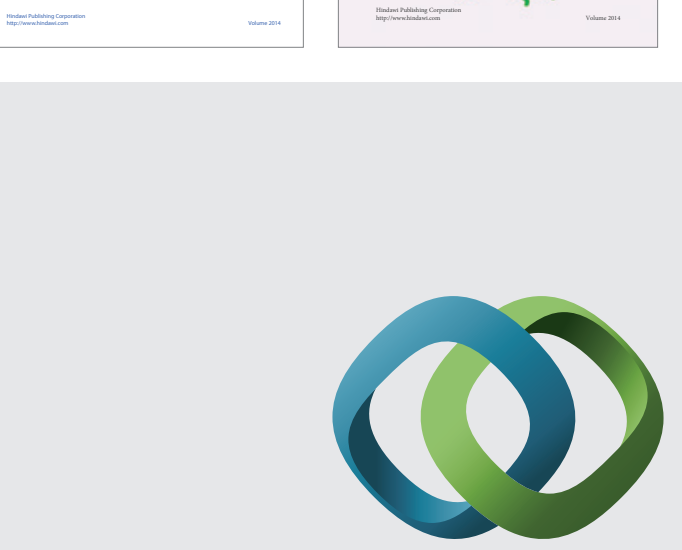

\section{Hindawi}

Submit your manuscripts at

http://www.hindawi.com
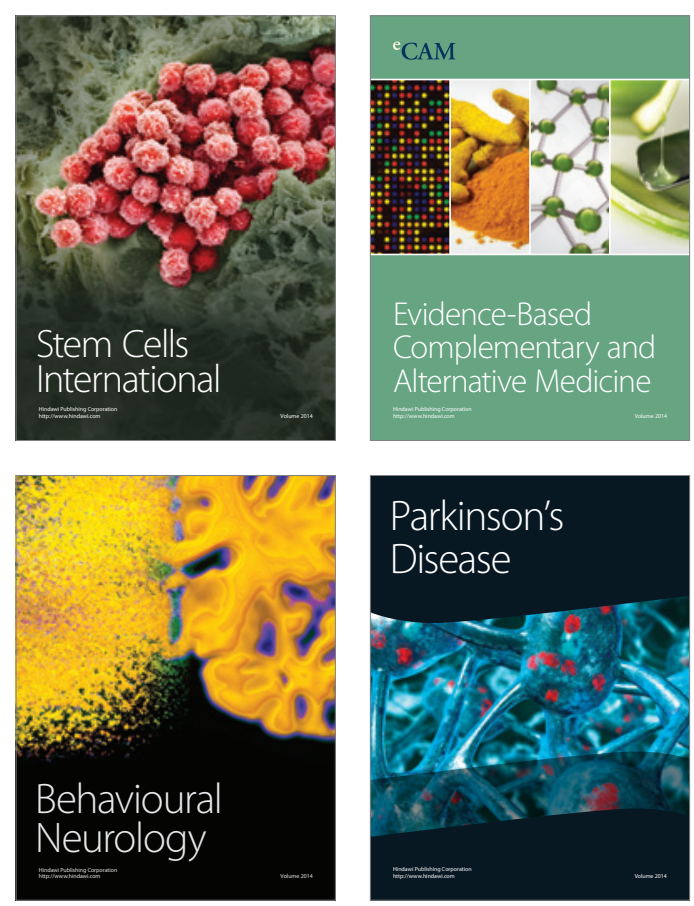

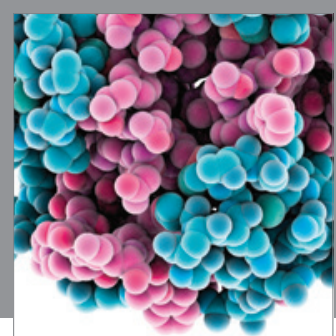

Journal of
Diabetes Research

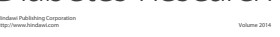

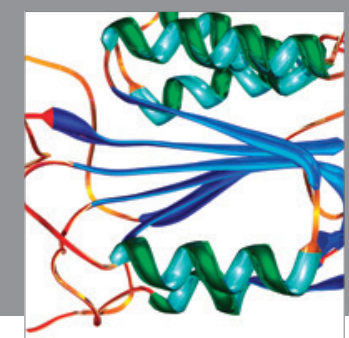

Disease Markers
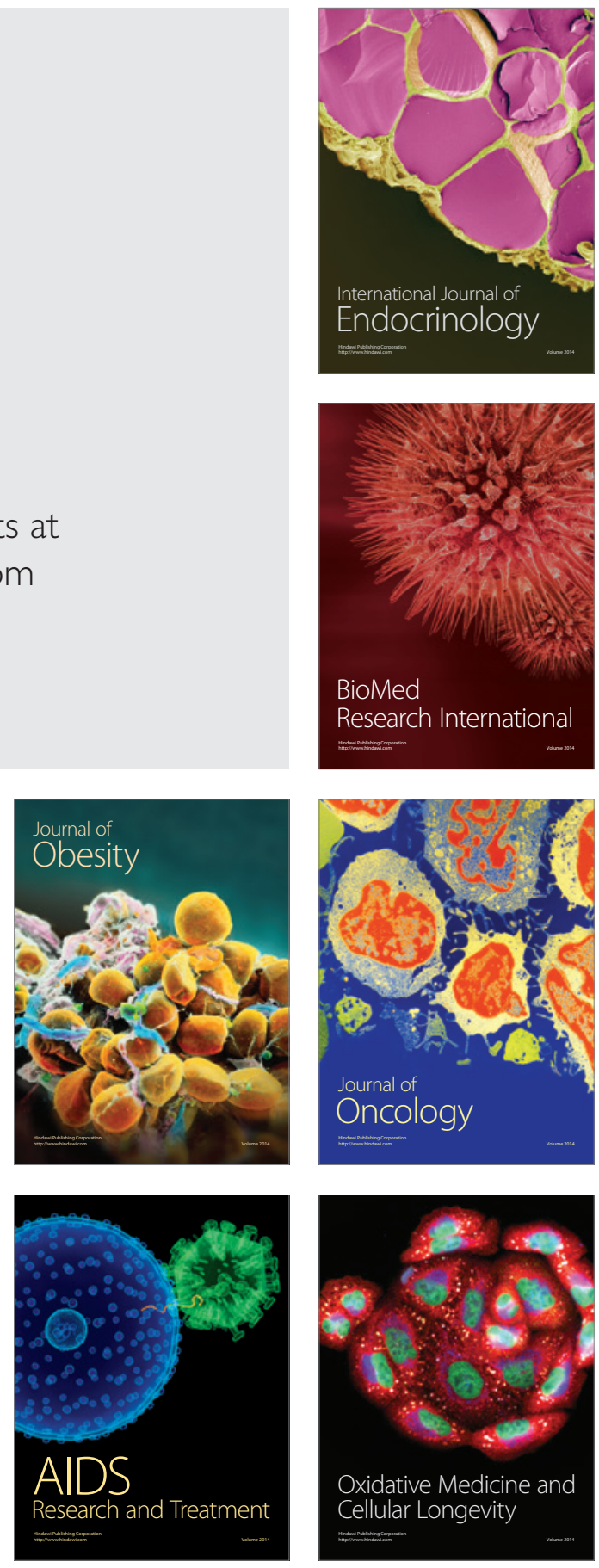